\title{
Characteristics of adolescents frequently restrained in acute psychiatric units in Norway: a nationwide study
}

\author{
Astrid Furre ${ }^{1,2^{*}}$, Ragnhild Sørum Falk ${ }^{3}$, Leiv Sandvik ${ }^{3}$, Svein Friis ${ }^{4}$, Maria Knutzen ${ }^{1}$ and Ketil Hanssen-Bauer ${ }^{5,6}$
}

\begin{abstract}
Background: The use of restraints in adolescent psychiatric settings requires particular professional, ethical, and legal considerations. The purpose of this study was to investigate whether the number of restraint episodes per patient was related to any of several characteristics of the adolescents.

Methods: In this nationwide study, we included all adolescents restrained during the period 2008-2010 ( $N=267)$ in Norwegian adolescent acute psychiatric inpatient units. They constitute $6.5 \%$ of the adolescents hospitalized in these units in the same period of time. We collected data on the number of restraint episodes they experienced during the study period; Poisson regression was then used to analyze the impact of gender, social, mental health, and treatment characteristics on the frequency of restraint. We developed a risk index for the likelihood of experiencing multiple restraint episodes.

Results: We found a skewed distribution of restraint episodes in which a small group (18\%) of restrained adolescents experienced a majority (77\%) of the restraint episodes. A large percentage of the restrained adolescents (36\%) experienced only one restraint episode. Risk factors for multiple restraint episodes were female gender, lower psychosocial functioning (Children's Global Assessment Scale below 35), more and longer admissions, and concomitant use of pharmacological restraint. Except for gender, we used these variables to develop a risk index that was moderately associated with multiple restraint episodes.

Conclusions: As a small group of patients accounted for a large percentage of the restraint episodes, future research should further investigate the reasons for and consequences of multiple restraint episodes in patients at acute adolescent psychiatric units, and evaluate preventive approaches targeted to reduce their risk for experiencing restraint.
\end{abstract}

Keywords: Adolescence, Health care research, Restraint, Inpatient

\section{Background}

Various types of restraint are used in psychiatric institutions to stop patients from harming themselves, others, or property, including mechanical restraints, seclusion, pharmacological restraints, and physical holding. The use of restraint is potentially harmful, and thus the overall objective is to minimize its use. The use of restraint against adolescents requires particular professional, ethi$\mathrm{cal}$, and legal considerations. The staff at many psychiatric

\footnotetext{
*Correspondence: astrid.furre@gmail.com; asfurr@ous-hf.no

${ }^{1}$ Division of Mental Health and Addiction, Centre for Forensic Psychiatry,

Oslo University Hospital, Oslo, Norway

Full list of author information is available at the end of the article
}

units for adolescents consider the use of restraint to be unavoidable to manage severe aggressive behavior. In child and adolescent psychiatry, the use of restraint (especially physical holding) has sometimes been considered therapeutic, even if there is little evidence of such benefit [1]. Rather, studies have shown that patients often experience restraint as coercion and trauma, and that this results in less trustful relations with the staff [2]. Some patients have described flashbacks from prior traumatic events during physical holding [2-4], and some are physically injured $[4,5]$. Use of restraint may also negatively influence the inpatient milieu [4]. 
A recent review of 49 studies of adult inpatient mental health services estimated that the prevalence of restraint was $3.8-20 \%$ and most frequently associated with male gender, younger age, foreign ethnicity, schizophrenia, involuntary admission, aggression or trying to abscond, and the presence of male staff [6]. Adult studies report substantial variability in the use of restraint between nations and hospitals [7-10]. Studies of restraint use in child and adolescent mental health services report relatively high rates, often at similar or higher levels compared to adult mental health services, and, again, the extent of the use varies considerably $[11,12]$. One Finnish national study reported that about $40 \%$ of the adolescent inpatients had been restrained in some way during their admission [13]. Another Finnish study reported that 27\% of the involuntary treatment periods in an 8-year period included the use of restraint and that there was considerable regional variation in the use of restraint [14]. In a previous paper based on the present study, we found that 267 (6.5\%) of the 4099 adolescents admitted (voluntarily or involuntarily) to acute psychiatric units in Norway in 2008-2010 were restrained [15].

Another important finding in previous studies of restrained adolescents is the skewed distributions of restraint episodes, with small proportions of adolescents accounting for large proportions of episodes, and large proportions of adolescents being restrained only once or twice [16-21]. Two of these studies found that adolescents who had been restrained many times were younger $[19,20]$, and four studies found that adolescents who had been restrained more than once had longer hospital stays $[17,18,20,21]$. One of these studies found that adolescents who had been restrained three or more times shared a particular profile: $67 \%$ had multiple admissions during the study period, all of them had a previous psychiatric hospitalization, and they were more likely to have lived in foster care, had special education, and a history of voicing suicidal ideation and attempting suicide [21]. Frequently restrained adolescents represent a specific challenge for the staff at inpatient psychiatric units, because the staff find the use of restraint necessary while acknowledging that there is the potential for physical and psychological harm with repeated episodes of restraint. Thus, for the sake of both the adolescents and the staff in such units, it is important to prevent the frequent restraint of adolescents. To our knowledge, no studies based on a nationwide sample have been published that identify the characteristics of adolescents who have experienced multiple restraint episodes in inpatient mental health services.

This paper presents data from a study on the use of restraint in acute psychiatric units for adolescents in Norway. We investigated whether the number of restraint episodes per patient was related to any of several characteristics of the adolescents. We also wanted to develop a risk index score based on our dataset to identify patients with higher risk for multiple restraint episodes.

\section{Methods}

\section{Setting}

We collected data from all of the Norwegian adolescent acute psychiatric inpatient units that are approved for involuntary admissions $(\mathrm{N}=16)$, which included a total of 126 beds (mean 7.4, SD 2.9, range 2-14). These units provide inpatient mental health care mainly for adolescents aged 13-17 years, but they accept admission of younger adolescents if needed. Some adolescents are more than 17 years old at the time of discharge. During the study period, all of the units accepted around-theclock emergency admissions. The units are distributed throughout Norway, and each unit has a uniquely defined catchment area. As a rule, drug-addicted adolescents are cared for by the child protection service. Three of the 16 acute psychiatric inpatient units were locked when needed, and the other 13 were permanently locked or had one permanently locked ward.

\section{Data collection}

We collected data on all of the inpatients in the included units who experienced restraint from January 1, 2008 through December 31, 2010. We collected the data retrospectively during a nine-month period from August 2011 to May 2012. The first author visited all of the institutions and collected data on restraint episodes, demographic characteristics, and clinical variables. Information about restraint episodes was collected from routinely used handwritten restraint protocols. Other data were collected from the electronic patient records. The total number of admitted adolescent patients during the study period was retrieved from the electronic patient administrative system at each unit.

\section{Definitions of restraint in the Norwegian Mental Health Care Act}

The Norwegian Mental Health Care Act regulates the practice of restraint procedures in Norway [22]. Staff members must consider less restrictive interventions first, and they cannot use restraint as a treatment. The following types of restraint may be used: (a) mechanical restraints, which inhibit the patient's freedom of movement, including belts and straps and clothing specially designed to prevent injury; (b) seclusion, which refers to detention for a short period of time behind a locked or closed door without a staff member present; (c) pharmacological restraint, which refers to single doses of medicines that have a short-term effect and are used to 
calm or sedate a patient; and (d) physical holding, which refers to any technique in which staff members physically restrain a patient without using tools. Mechanical restraints and locked seclusion are not allowed for patients under the age of 16 . Restraint can be used during either voluntary or compulsory admissions. All psychiatric institutions in Norway are obligated by law to have a restraint protocol in which each restraint episode is registered. The protocol describes the type and duration of the restraint and the reason for its use. Independent and authorized control commissions regularly checks all registrations in these protocols.

In this study, we did not include episodes of restraint that were needed for compulsory feeding in cases of severe anorexia (1896 restraint episodes distributed across 21 patients). The Norwegian Mental Health Act also regulates the use of compulsory feeding for patients with anorexia. These episodes are often included in the restraint protocols because wards may use mechanical restraints or physical holding to conduct forced feeding. However, whether or not these episodes are included in the protocols varies between wards.

\section{Data and variables}

The dependent variable was the number of restraint episodes for each patient from all the admissions during the whole study period. The number of restraint episodes was categorized as $1,2-4,5-9$, and $\geq 10$. For adolescent patients with more than one admission in the three-year period, we collected data on the patient's social and mental health characteristics from the most recent admission.

Social characteristics. We defined immigrant background as having two foreign-born parents, and coded this Yes or No. The variable living arrangement at the time of the most recent admission was coded in four categories: living with both parents (biological or adoptive), living with one parent (with or without stepparent), living in foster care or institution, and other. The variable current involvement with the child protection service was coded Yes or No.

Mental health characteristics. The local clinical teams assessed the adolescent patients' conditions and coded their main psychiatric disorder using the Axis One (clinical psychiatric syndromes) in the multiaxial ICD-10 classification of child and adolescent psychiatric disorders from the World Health Organization [23]. Using this information, we grouped the adolescent patients into one of five categories based on their most recent admission (the ICD-10 codes are in parentheses): (1) No Axis One disorder; (2) psychotic (F20-29) or pervasive developmental disorder (F84); (3) manic episode or bipolar affective disorder (F30, F31.0-F31.2, F31.6-31.9); (4) internalizing disorder (depression F31.3, F32-33; anxiety F40-41, F93,
F94; OCD F42; stress related F43; dissociative F44); (5) externalizing disorders (substance use F10, F12, F19; personality F60, F69; hyperkinetic F90; conduct F91-92; tics F95). Global psychosocial functioning was routinely rated by the clinicians at admission using the Children's Global Assessment Scale (CGAS) [24]. We used the CGAS score from each patient's most recent admission. The CGAS measures general functioning, with scores ranging from 1 (needs constant supervision) to 100 (superior functioning). We divided CGAS scores into three groups (tertiles): $1-34,35-44$, and $45-75$. We did not measure the interrater reliability of the CGAS for this study. However, the interrater reliability of the CGAS in routine use was found to be moderate (intraclass correlation coefficient, .61) in a large study of clinicians in Norwegian outpatient child and adolescent mental health services [25].

Treatment characteristics. We divided the number of admissions in the study period into three groups (tertiles): $1,2-3$, and $\geq 4$ admissions. We defined the length of admission as the number of days for the most recent admission and we divided this into three groups (tertiles): $1-4,5-21$, and $\geq 22$ days. We coded involuntary admission as Yes if the patient was involuntarily admitted during the study period. We defined concomitant use of restraint as the use of pharmacological restraint in combination with any of the other types of restraint, and it was coded Yes when it occurred.

We developed a risk index score using the patient characteristic variables that were significantly associated with the number of restraint episodes (as indicated by the multivariate regression analysis). The categories for the variables were scored as 0,1 , or 2 (depending upon the number of possible response categories), with higher scores representing a stronger positive association with the number of restraint episodes. These scores were summed to make the risk index score. Because of the retrospective design of this study, and the fact that some of the variables required the completion of inpatient care, the prospective use of this risk index score at the patient level is limited. However, it may be useful to compare groups of adolescents admitted to inpatient care.

\section{Statistical analysis}

Descriptive statistics are presented as frequencies and percentages. Zero-truncated Poisson regression analysis was applied to analyze the impact of gender, social, mental health, and treatment characteristics on the number of restraint episodes. We did not include age in our regression analyses; because each patient's date of birth and exact age at the date of admission were unknown (only the age attained during the calendar year was available). In addition, adolescents must be at least 16 years old to be involuntarily admitted and to be restrained 
by mechanical means or seclusion. We performed univariate analyses for the independent variables: gender, immigrant background, living arrangement, current involvement with the child protection service, main psychiatric disorder, global psychosocial functioning (CGAS score), number of admissions in the study period, length of admission, involuntary admission, and concomitant use of restraint. Variables with $p<.20$ in the analysis were selected for inclusion in the multivariate model. Variables that were not statistically significant $(p \geq .05)$ in the multivariate analysis were deleted (largest $p$ values first) until all of the remaining variables were significantly associated with the outcome. We used robust standard errors for the parameter estimates, as recommended by Cameron and Trivedi [26]. The effects are presented as incidence rate ratios (IRR) with 95\% confidence intervals and $\mathrm{p}$ values. We tested the final model for multicollinearity by calculating the variance inflation factors for each of the independent variables. Estimated model fit is presented as pseudo $R^{2}$ (explained variance). Because of the large number of missing values in CGAS (70 adolescents had no information), we reran the analyses omitting CGAS from the model to check for selection bias. We used the Goodman and Kruskal's rank correlation statistics to measure the strength of association between the risk index score and the observed number of restraint episodes.

A $\mathrm{p}$ value $<.05$ was regarded as statistically significant. All statistical analyses were conducted using SPSS (version 18.0) and Stata [27, 28].

\section{Results}

\section{Study sample}

The sample comprised 267 adolescents who had experienced one or more restraint episodes during the 3-year study period (2008-2010). This group of 267 adolescents constitutes $6.5 \%$ of the 4099 adolescents admitted one or more times to the units in the same period. The mean age of the sample was 16.0 years (SD 1.8, range 10-21), 158 (59\%) of whom were female. Seven of the adolescents were 10 or 11 years old, and eight were 19-21 years old. Eighteen (7\%) of the adolescents experienced concomitant pharmacological restraint, most of them during episodes of physical holding. One adolescent was pharmacologically restrained in combination with seclusion and three in combination with mechanical restraint. The sample is described in detail in Table 1.

\section{Distribution of restraint episodes}

The adolescents in our sample experienced 2277 restraint episodes $(78.7 \%$ of these were psychical holding, $13.4 \%$ mechanical restraint, $5.9 \%$ seclusion, and $1.6 \%$ pharmacological restraint) during the study period. The median number of restraint episodes per patient was two (range $1-171)$. Figure 1 shows that $97(36 \%)$ of the adolescents were only restrained once, 88 (33\%) 2-4 times, 35 (13\%) 5-9 times, and 47 (18\%) 10 times or more. This latter group accounted for 1762 (77\%) of the restraint episodes.

\section{Regression analysis}

The results from the regression analysis are presented in Table 2. The univariate analyses showed that having an Axis One disorder, lower global psychosocial functioning (CGAS below 35), more admissions in study period, an admission of 22 days or longer, and concomitant use of restraint were associated with more restraint episodes, whereas living with one parent was associated with fewer restraint episodes than living with both parents. The other variables were not significantly associated with the number of restraint episodes.

The multivariate analysis indicated that being female, lower global psychosocial functioning (CGAS below 35), more admissions in the study period, an admission of 22 days or longer, and concomitant use of restraint were associated with more restraint episodes. We found no evidence of multicollinearity in the multivariate regression analysis. Omitting CGAS from the model gave similar results (data not shown). The $\mathrm{R}^{2}$ of the adjusted model was $31.9 \%$.

\section{Risk index score}

The five significant variables from the multivariate model were considered for inclusion in the risk index score (risk for more restraint episodes). However, we did not include gender in the risk index score, because the finding that females have a greater risk of restraint episodes is not generally supported in the literature, and this variable had the lowest IRR. The remaining four variables were included in the risk index score, and the scoring system is shown in Table 3. The categories for the risk index score were tabulated based on the number of restraint episodes per patient (Table 4). The likelihood of experiencing 10 or more restraint episodes increased from $0 \%$ when the risk index score was $\leq 1$ to $35 \%$ when the risk index score was $\geq 4$. The Goodman and Kruskal's correlation was .35.

\section{Discussion}

We found a skewed distribution of restraint episodes. A small group of restrained patients (18\%) who were restrained more than 10 times accounted for the majority (77\%) of the restraint episodes. The multivariate analysis revealed that the adolescents who were likely to experience more restraint episodes were female, with lower global psychosocial functioning, more and longer admissions, and those who had experienced concomitant use of restraint. Four of these five variables (excluding gender) 
Table 1 Relationship between patient characteristics and the number of restraint episodes

\begin{tabular}{|c|c|c|c|c|c|}
\hline & \multicolumn{4}{|c|}{ Number of restraint episodes } & \multirow[t]{3}{*}{$\mathbf{N}$} \\
\hline & 1 & $2-4$ & $5-9$ & $\geq 10$ & \\
\hline & $\mathrm{N}(\%)$ & $\mathrm{N}(\%)$ & $\mathrm{N}(\%)$ & $\mathrm{N}(\%)$ & \\
\hline Total sample (patients) & $97(36)$ & $88(33)$ & $35(13)$ & $47(18)$ & 267 \\
\hline \multicolumn{6}{|l|}{ Gender } \\
\hline Female & $59(37)$ & $49(31)$ & $17(11)$ & $33(21)$ & 158 \\
\hline Male & $38(35)$ & $39(36)$ & $18(17)$ & $14(13)$ & 109 \\
\hline \multicolumn{6}{|l|}{ Social characteristics } \\
\hline \multicolumn{6}{|l|}{ Immigrant background } \\
\hline Yes & $11(31)$ & $15(43)$ & $4(11)$ & $5(14)$ & 35 \\
\hline No & $79(37)$ & $69(32)$ & $27(13)$ & $38(18)$ & 213 \\
\hline Missing & $7(37)$ & $4(21)$ & $4(21)$ & $4(21)$ & 19 \\
\hline \multicolumn{6}{|l|}{ Living arrangement } \\
\hline With both parents & $32(37)$ & $26(30)$ & $8(9)$ & $21(24)$ & 87 \\
\hline With one parent (with or without stepparent) & $26(41)$ & $23(37)$ & $9(14)$ & $5(8)$ & 63 \\
\hline Foster care or institution & $36(34)$ & $36(34)$ & $16(15)$ & $19(18)$ & 107 \\
\hline Other & $1(14)$ & $3(43)$ & $1(14)$ & $2(29)$ & 7 \\
\hline Missing & $2(67)$ & $0(0)$ & $1(33)$ & $0(0)$ & 3 \\
\hline \multicolumn{6}{|l|}{ Current involvement with the child protection service } \\
\hline Yes & $40(33)$ & $43(35)$ & $19(15)$ & $21(17)$ & 123 \\
\hline No & $37(35)$ & $33(31)$ & $12(11)$ & $23(22)$ & 105 \\
\hline Missing & $20(51)$ & $12(31)$ & $4(10)$ & $3(8)$ & 39 \\
\hline \multicolumn{6}{|l|}{ Mental health characteristics } \\
\hline \multicolumn{6}{|l|}{ Main psychiatric disorder (Axis One onlya) } \\
\hline No Axis One disorder & $23(58)$ & $9(22)$ & $5(13)$ & $3(8)$ & 40 \\
\hline Psychotic or pervasive developmental disorder & $19(50)$ & $9(24)$ & $2(5)$ & $8(21)$ & 38 \\
\hline Manic episode or bipolar affective disorder & $4(21)$ & $7(37)$ & $5(26)$ & $3(16)$ & 19 \\
\hline Internalizing disorder & $28(29)$ & $33(35)$ & $15(15)$ & $19(20)$ & 95 \\
\hline Externalizing disorder & $23(31)$ & $30(40)$ & $8(11)$ & $14(19)$ & 75 \\
\hline \multicolumn{6}{|l|}{$\mathrm{CGAS}^{\mathrm{b}}$} \\
\hline $1-34$ & $15(23)$ & $24(37)$ & $8(12)$ & $18(28)$ & 65 \\
\hline $35-44$ & $23(37)$ & $22(35)$ & $8(13)$ & $9(15)$ & 62 \\
\hline $45-75$ & $30(43)$ & $19(27)$ & $8(11)$ & $13(19)$ & 70 \\
\hline Missing & $29(41)$ & $23(33)$ & $11(16)$ & $7(10)$ & 70 \\
\hline \multicolumn{6}{|l|}{ Treatment characteristics } \\
\hline \multicolumn{6}{|l|}{ Number of admissions in the study period } \\
\hline 1 & $46(46)$ & $39(39)$ & $8(8)$ & $8(8)$ & 101 \\
\hline $2-3$ & $27(31)$ & $30(34)$ & $15(17)$ & $15(17)$ & 87 \\
\hline $4-19$ & $24(30)$ & $19(24)$ & $12(15)$ & $24(30)$ & 79 \\
\hline \multicolumn{6}{|l|}{ Length of admission (days) } \\
\hline $0-4$ & $41(52)$ & $23(29)$ & $8(10)$ & $7(9)$ & 79 \\
\hline $5-21$ & $31(30)$ & $38(37)$ & $18(17)$ & $17(16)$ & 104 \\
\hline $22-425$ & $25(30)$ & $27(32)$ & $9(11)$ & $23(27)$ & 84 \\
\hline \multicolumn{6}{|l|}{ Involuntary referral } \\
\hline Yes & $28(27)$ & $30(29)$ & $17(16)$ & $29(28)$ & 104 \\
\hline No & $66(42)$ & $54(35)$ & $18(12)$ & $18(12)$ & 156 \\
\hline Missing & $3(43)$ & $4(57)$ & $0(0)$ & $0(0)$ & 7 \\
\hline
\end{tabular}




\begin{tabular}{|c|c|c|c|c|c|}
\hline & \multicolumn{4}{|c|}{ Number of restraint episodes } & \multirow[t]{3}{*}{$\mathbf{N}$} \\
\hline & 1 & $2-4$ & $5-9$ & $\geq 10$ & \\
\hline & $\mathrm{N}(\%)$ & $\mathrm{N}(\%)$ & $\mathrm{N}(\%)$ & $\mathrm{N}(\%)$ & \\
\hline \multicolumn{6}{|c|}{ Concomitant use of restraint } \\
\hline Yes & $3(17)$ & $5(28)$ & $2(11)$ & $8(44)$ & 18 \\
\hline No & $94(38)$ & $83(33)$ & $33(13)$ & $39(16)$ & 249 \\
\hline
\end{tabular}

a Axis One is the clinical psychiatric syndromes [23]

b CGAS Children's Global Assessment Scale (global psychosocial functioning)

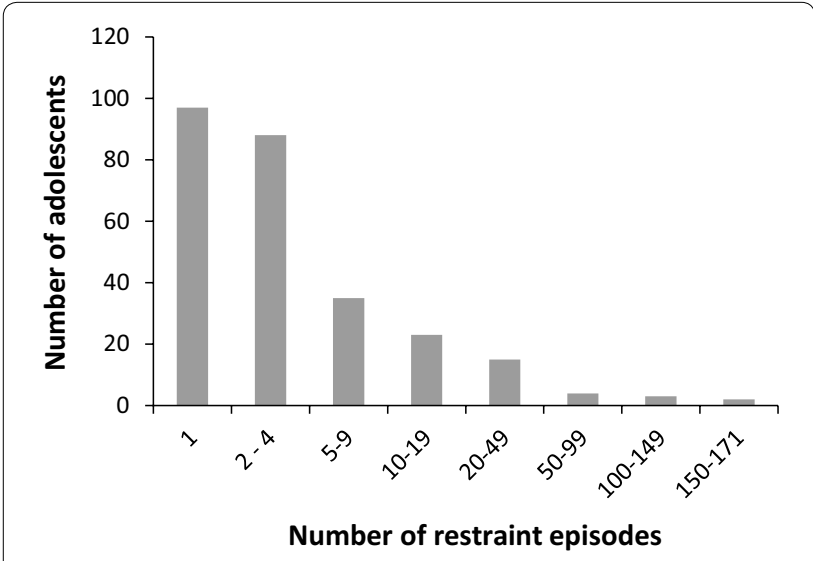

Fig. 1 Distribution of 2277 restraint episodes among 267 adolescents

were used to construct a risk index score, with higher scores indicating a greater risk of restraint episodes.

Earlier studies have also found highly skewed frequencies of restraint. One US study found that seven (1.7\%) out of 408 adolescents experienced $56.6 \%$ of 1349 aggressive episodes requiring an intervention [17]. A study on an adolescent inpatient unit reported that $7.4 \%$ of the secluded patients experienced $81 \%$ of the seclusion episodes, and two of these patients (1.3\%) experienced $45 \%$ of the seclusion episodes [16]. Another large retrospective study collected data on all restraint episodes during 7.5 years in a US psychiatric hospital for children, adolescents, and adults with severe mental illness. They found that 20,10 , and $1 \%$ of the most-often restrained patients experienced 75,61 , and $21 \%$ of the restraint episodes, respectively [29].

\section{Gender}

We found that females had a greater risk of multiple restraint episodes. A study of adolescents in two US residential treatment centers found that adolescents with moderate and high levels of seclusion and restraint were more often black and/or female [30,31]. However, there are studies of adolescents with different findings that give a more inconclusive picture of the evidence $[17,18,21$, 32]. A recent adult study from Norway found slightly more females than males among the frequently restrained patients, but the difference was not statistically significant [33]. A reason for the inconclusive picture may be that different nations treat different adolescent populations in their psychiatric inpatient units. There may also be different pathways to multiple restraint episodes for female and male adolescents which are not controlled for in the different studies.

\section{Social characteristics}

None of the social characteristics (immigrant background, living arrangement or current involvement with the child protection service) increased the risk of multiple restraint episodes in the multivariate model. Immigrant background was a significant predictor of being restrained compared to not being restrained in our previous paper, while the other two social characteristics were not [34]. We have not found other studies that analyzed these characteristics as risk factors for multiple restraint episodes.

\section{Mental health characteristics}

We found that that lower global psychosocial functioning (CGAS below 35) was associated with more restraint episodes. One study has reported that adolescent inpatients with more than 50 aggressive episodes requiring intervention had a lower mean global psychosocial functioning at admission [17].

Having an Axis One psychiatric disorder was not a significant risk factor in the multivariate analyses. A previous study of female adolescents found that externalizing or internalizing disorders (versus no diagnosis) predicted restraint episodes, even in a multivariate regression analysis [35]. Another study did not include diagnoses in the multivariate analyses because of high multicollinearity with admission status (court ordered, physician's emergency certificate, or voluntary) [36]. 
Table 2 Risk factors for more frequent restraint episodes

\begin{tabular}{|c|c|c|c|c|c|c|}
\hline & \multicolumn{3}{|c|}{ Unadjusted model } & \multicolumn{3}{|c|}{ Adjusted model } \\
\hline & $\mathrm{IRR}^{\mathbf{b}}$ & $95 \% \mathrm{Cl}^{\mathrm{a}}$ & $p$ & $\mathrm{IRR}^{\mathbf{b}}$ & $95 \% \mathrm{Cl}^{\mathrm{a}}$ & $p$ \\
\hline \multicolumn{7}{|l|}{ Gender (ref: male) } \\
\hline Female & 1.69 & $.99-2.90$ & .055 & 2.04 & $1.1-3.74$ & .021 \\
\hline \multicolumn{7}{|l|}{ Social characteristics } \\
\hline \multicolumn{7}{|l|}{ Immigrant background (ref: yes) } \\
\hline No & 1.09 & $.37-3.18$ & .876 & & & \\
\hline \multicolumn{7}{|l|}{ Living arrangement (ref: with both parents) } \\
\hline With one parent (with or without stepparent) & .42 & $.19-.94$ & .034 & & & \\
\hline Foster care or institution & .81 & $.42-1.57$ & .530 & & & \\
\hline Other & 1.18 & $.40-3.46$ & .767 & & & \\
\hline \multicolumn{7}{|c|}{ Current involvement with the child protection service (ref: no) } \\
\hline Yes & .85 & $.46-1.57$ & .610 & & & \\
\hline \multicolumn{7}{|l|}{ Mental health characteristics } \\
\hline \multicolumn{7}{|l|}{ Main psychiatric disorder (ref: no Axis One disorder) } \\
\hline Psychotic or pervasive developmental disorder & 4.08 & $1.50-11.09$ & .006 & & & \\
\hline Manic episode or bipolar affective disorder & 6.45 & $1.93-21.52$ & .002 & & & \\
\hline Internalizing disorder & 3.85 & $2.09-21.52$ & $<.001$ & & & \\
\hline Externalizing disorders & 3.74 & $1.99-7.05$ & $<.001$ & & & \\
\hline \multicolumn{7}{|l|}{ Global psychosocial functioning } \\
\hline \multicolumn{7}{|l|}{ CGAS (ref 45-75) } \\
\hline $1-34$ & 2.35 & $1.27-4.35$ & .006 & 2.59 & $1.34-5.04$ & .005 \\
\hline $35-44$ & 1.48 & $.67-3.26$ & .334 & 1.70 & $.71-4.11$ & .236 \\
\hline \multicolumn{7}{|l|}{ Treatment characteristics } \\
\hline \multicolumn{7}{|l|}{ Number of admissions in the study period (ref: 1) } \\
\hline $2-3$ & 2.33 & $1.07-5.09$ & .034 & 2.32 & $1.08-4.95$ & .030 \\
\hline $4-19$ & 2.78 & $1.40-5.51$ & .003 & 3.28 & $1.76-6.13$ & $<.001$ \\
\hline \multicolumn{7}{|l|}{ Length of last admission in days (ref: $1-4$ ) } \\
\hline $5-21$ & 1.12 & $.55-2.28$ & .757 & 1.02 & $.51-2.03$ & .956 \\
\hline $22-425$ & 2.43 & $1.14-5.20$ & .022 & 2.63 & $1.21-5.66$ & .014 \\
\hline \multicolumn{7}{|l|}{ Involuntary referral (ref: no) } \\
\hline Yes & 1.70 & $.93-3.08$ & .083 & & & \\
\hline \multicolumn{7}{|l|}{ Concomitant use of restraint (ref: no) } \\
\hline Yes & 3.97 & $2.01-7.84$ & $<.001$ & 3.44 & $1.90-6.22$ & $<.001$ \\
\hline
\end{tabular}

Based on zero-truncated Poisson regression analysis

a $\mathrm{Cl}$ confidence interval

b IRR incidence rate ratio

\section{Treatment characteristics}

Our finding that more and longer admissions are associated with a greater number of restraint episodes has been reported in three previous US studies [17, 21,35].

In the present study, involuntary admission was not a risk factor for multiple restraint episodes. One US study found that admission status was the strongest and most consistent predictor of frequent restraint [36]. However, their admission procedures (court ordered, physician's emergency certificate, or voluntary) differ from those in Norway.
In our study, $7 \%$ of the restrained adolescents experienced concomitant use of restraint. A recent Finnish study found that $8.5 \%$ of the mechanically restrained adolescents received intramuscular medication during the restraint episode [37]. However, they did not analyze whether this concomitant use was a predictor for more restraint episodes. In the present study, we found that the use of concomitant restraint was a strong risk factor for frequent restraint episodes. As far as we know, this has not been reported previously. One rationale for using concomitant restraint is that it shortens the duration of 
Table 3 Scoring system for the risk index score

\begin{tabular}{lll}
\hline Variable & Results & Scores \\
\hline Global psychosocial functioning (CGAS) & $1-34$ & 2 \\
& $35-44$ & 1 \\
Number of admissions & $\geq 45$ & 0 \\
& 1 & 0 \\
Length of admission (days) & $2-3$ & 1 \\
& $\geq 4$ & 2 \\
Concomitant use of restraint & $1-4$ & 0 \\
& $5-21$ & 1 \\
& $\geq 22$ & 2 \\
& No & 0 \\
\hline
\end{tabular}

Possible range of the risk index score: $0-7$

\begin{tabular}{llllll}
$\begin{array}{l}\text { Table } 4 \\
\text { Relationship } \\
\text { and restraint episodes }\end{array}$ & between & risk & index & score \\
\hline Risk index score & $\mathbf{N}(\%)$ & \multicolumn{4}{l}{ Number of restraint episodes } \\
\cline { 3 - 7 } & & $\mathbf{0 - 1}$ & $\mathbf{2 - 4}$ & $\mathbf{5 - 9}$ & $\mathbf{2 1 0}$ \\
& & $\mathbf{n ( \% )}$ & $\mathbf{n}(\%)$ & $\mathbf{n}(\%)$ & $\mathbf{n ( \% )}$ \\
\hline $0-1$ & $28(100)$ & $15(54)$ & $10(36)$ & $3(11)$ & $0(0)$ \\
2 & $38(100)$ & $16(42)$ & $11(29)$ & $5(13)$ & $6(16)$ \\
3 & $63(100)$ & $23(37)$ & $22(35)$ & $8(13)$ & $10(16)$ \\
$4-7$ & $68(100)$ & $14(21)$ & $22(32)$ & $8(12)$ & $24(35)$ \\
\hline
\end{tabular}

Goodman and Kruskal's gamma is .35

Data from $\mathrm{N}=197$ adolescents with valid Information for all four of the variables used for the risk index score

restraint [17], but we have not found any studies that document this effect. Our finding indicates that this group deserves special attention to prevent the use of restraint in clinical practice.

\section{Risk index score}

The risk index score that we developed was moderately associated with a larger number of restraint episodes. The risk index score is easy to calculate and it may be useful in clinical practice. However, these results should be replicated in other samples before the risk index score can be implemented in clinical use.

\section{Strengths and limitations}

A methodological strength of our study relative to previous studies is the highly representative national sample. We used a 3-year cohort of all of the adolescent inpatients who had experienced restraint in all of the acute psychiatric units in Norway. In addition, we had access to data for all of the registered restraint episodes they experienced during that period.
One limitation of this study is the retrospective design, which leaves us unable to determine the reliability of the clinical data, such as the main psychiatric disorders, the CGAS scores, and the reasons for the use of restraint. A second limitation is the lack of a reliability test of the data extraction. We are not aware whether our findings had been different if we had collected data on social and mental health characteristics from another admission than the most recent, i.e. the first.

\section{Conclusion}

Based on a large and representative national dataset, a notable finding in this study is the skewed distribution of restraint episodes. A small group of frequently restrained adolescent patients (18\%) accounted for a large percentage $(77 \%)$ of the restraint episodes. Conversely, a large percentage of the restrained adolescents $(36 \%)$ were only restrained once. Factors associated with more restraint episodes were female gender, low global psychosocial functioning, longer and more admissions, and concomitant use of pharmacological restraint. A risk index score based on four variables was moderately associated with more restraint episodes in our sample. If these findings are replicated in other samples, the identification of patients at high risk of multiple restraint episodes may inform the development of interventions to reduce the use of restraint. The effects of such interventions should be evaluated in well-designed future research trials.

\section{Abbreviation \\ CGAS: Children's Global Assessment Scale.}

\section{Authors' contributions}

AF designed the study, collected the data and drafted the main manuscript versions. All authors provided comments on the different versions. RSF and LS conducted the statistical analyses. All authors read and approved the final manuscript.

\section{Author details \\ ${ }^{1}$ Division of Mental Health and Addiction, Centre for Forensic Psychiatry, Oslo University Hospital, Oslo, Norway. ${ }^{2}$ Centre for Child and Adolescent Mental Health, Eastern and Southern Norway, Oslo, Norway. ${ }^{3}$ Oslo Centre for Epide- miology and Biostatistics, Oslo University Hospital, Oslo, Norway. ${ }^{4}$ Division of Mental Health and Addiction, Department of Research and Development, Oslo University Hospital, Oslo, Norway. ${ }^{5}$ Division of Mental Health Services, Akershus University Hospital, Lorenskog, Norway. ${ }^{6}$ Division of Health Service Research and Psychiatry, University of Oslo, Oslo, Norway.}

Acknowledgements

The authors thank Dr. Sonja Heyerdahl for contributions to the study design.

\section{Competing interests}

The authors declare that they have no competing interests.

\section{Availability of data and materials}

Data are not in the public domain but requests can be sent to the corresponding author for consideration. 


\section{Ethics approval and consent to participate}

The South and East Regional Committee for Medical and Health Research Ethics, Norway (project No 08/5835) and the Privacy Protection Officer at Oslo University Hospital, Norway, approved this study. They approved that we did not ask participants for consent to participate or consent to publish.

Received: 6 September 2016 Accepted: 6 December 2016 Published online: 12 January 2017

\section{References}

1. Day DM. Examining the therapeutic utility of restraints and seclusion with children and youth: the role of theory and research in practice. Am J Orthopsychiatry. 2002;72(2):266-78.

2. Kalogjera IJ, Bedi A, Watson WN, Meyer AD. Impact of therapeutic management on use of seclusion and restraint with disruptive adolescent inpatients. Hosp Community Psychiatry. 1989;40(3):280-5.

3. Mohr WK, Petti TA, Mohr BD. Adverse effects associated with physical restraint. Can J Psychiatry. 2003;48(5):330-7.

4. LeBel J, Stromberg N, Duckworth K, Kerzner J, Goldstein R, Weeks M, et al. Child and adolescent inpatient restraint reduction: a state initiative to promote strength-based care. J Am Acad Child Adolesc Psychiatry. 2004:43(1):37-45

5. Caldwell B, Albert C, Azeem MW, Beck S, Cocoros D, Cocoros T, et al. Successful seclusion and restraint prevention effort in child and adolescent programs. J Psychosoc Nurs Ment Health Serv. 2014;52(11):30-8.

6. Beghi M, Peroni F, Gabola P, Rossetti A, Cornaggia CM. Prevalence and risk factors for the use of restraint in psychiatry: a systematic review. Riv Psichiatr. 2013;48(1):10-22.

7. Bowers L, van der Werf B, Vokkolainen A, Muir-Cochrane E, Allan T, Alexander J. International variation in containment measures for disturbed psychiatric inpatients: a comparative questionnaire survey. Int J Nurs Stud. 2007;44(3):357-64.

8. Kalisova L, Raboch J, Nawka A, Sampogna G, Cihal L, Kallert TW, et al. Do patient and ward-related characteristics influence the use of coercive measures? Results from the EUNOMIA international study. Soc Psychiatry Psychiatr Epidemiol. 2014;49(10):1619-29.

9. Raboch J, Kalisova L, Nawka A, Kitzlerova E, Onchev G, Karastergiou A, et al. Use of coercive measures during involuntary hospitalization: findings from ten European countries. Psychiatr Serv. 2010;61(10):1012-7.

10. Way BB, Banks SM. Use of seclusion and restraint in public psychiatric hospitals: patient characteristics and facility effects. Hosp Community Psychiatry. 1990;41(1):75-81.

11. LeBel J, Huckshorn KA, Caldwell B. Restraint use in residential programs: why are best practices ignored? Child Welfare. 2010;89(2):169-87.

12. De Hert M, Dirix N, Demunter H, Correll CU. Prevalence and correlates of seclusion and restraint use in children and adolescents: a systematic review. Eur Child Adolesc Psychiatry. 2011;20(5):221-30.

13. Sourander A, Ellila $H$, Valimaki $M$, Piha J. Use of holding, restraints, seclusion and time-out in child and adolescent psychiatric in-patient treatment. Eur Child Adolesc Psychiatry. 2002;11(4):162-7.

14. Siponen S, Maritta V, Riittakerttu KH. The use of coercive measures in adolescent psychiatric inpatient treatment: a nation-wide register study. Soc Psychiatry Psychiatr Epidemiol. 2012;47(9):1401-8.

15. Furre A, Sandvik L, Friis S, Knutzen M, Hanssen-Bauer K. A nationwide study of why and how acute adolescent psychiatric units use restraint. Psychiatry Res. 2016;237:60-6.

16. Angold A, Pickles A. Seclusion on an adolescent unit. J Child Psychol Psychiatry. 1993;34(6):975-89.

17. Baeza I, Correll CU, Saito E, Amanbekova D, Ramani M, Kapoor S, et al. Frequency, characteristics and management of adolescent inpatient aggression. J Child Adolesc Psychopharmacol. 2013;23(4):271-81.
18. Earle KA, Forquer SL. Use of seclusion with children and adolescents in public psychiatric hospitals. Am J Orthopsychiatry. 1995;65(2):238-44.

19. Goren S, Singh NN, Best AM. The aggression-coercion cycle: use of seclusion and restraint in a child psychiatric hospital. J Child Fam Stud. 1993;2(1):61-73.

20. Pogge DL, Pappalardo S, Buccolo M, Harvey PD. Prevalence and precursors of the use of restraint and seclusion in a private psychiatric hospital: comparison of child and adolescent patients. Adm Policy Ment Health. 2013:40(3):224-31.

21. Delaney KR, Fogg L. Patient characteristics and setting variables related to use of restraint on four inpatient psychiatric units for youths. Psychiatr Serv. 2005;56(2):186-92.

22. Act No. 62 of 2 July 1999 relating to the provision and implementation of mental health care, with later amendments. http://www.ub.uio.no/ujur/ ulovdata/lov-19990702-062-eng.pdf.

23. World Health Organization. Multiaxial classification of child and adolescent psychiatric disorders: The ICD-10 classification of mental and behavioural disorders in children and adolescents. Cambridge: Cambridge University Press; 1996

24. Shaffer D, Gould MS, Brasic J, Ambrosini P, Fisher P, Bird H, et al. A children's global assessment scale (CGAS). Arch Gen Psychiatry. 1983:40(11):1228-31.

25. Hanssen-Bauer K, Aalen OO, Ruud T, Heyerdahl S. Inter-rater reliability of clinician-rated outcome measures in child and adolescent mental health services. Adm Policy Ment Health. 2007;34(6):504-12.

26. Cameron AC, Trivedi PK. Microeconometrics using Stata: Stata Press; 2009

27. SPSS Inc. PASW Statistics for Windows. In: Version 18.0 edn. Chicago: SPSS Inc:;2009.

28. StataCorp. Stata Statistical Software: Release 13. In: College Station edn. Tx: StataCorp LP; 2013.

29. Whitehead PD, Liljeros F. Heavy-tailed distribution of seclusion and restraint episodes in a state psychiatric hospital. J Am Acad Psychiatry Law. 2011:39(1):93-9.

30. Miller L, Riddle MA, Pruitt D, Zachik A, dosReis S. Antipsychotic treatment patterns and aggressive behavior among adolescents in residential facilities. J Behav Health Serv Res. 2013;40(1):97-110.

31. dosReis S, McCulloch JR, Colantuoni E, Barnett SR, Pruitt D, Zachik A, et al. Early identification of seclusion and restraint patterns during adolescents' course of residential treatment. Resid Treat Child Youth. 2010;27(3):160-74

32. Martin A, Krieg H, Esposito F, Stubbe D, Cardona L. Reduction of restraint and seclusion through collaborative problem solving: a five-year prospective inpatient study. Psychiatr Serv. 2008;59(12):1406-12.

33. Knutzen M, Bjørkly S, Eidhammer G, Lorentzen S, Mjøsund NH, Opjordsmoen $\mathrm{S}$, et al. Characteristics of patients frequently subjected to pharmacological and mechanical restraint-a register study in three Norwegian acute psychiatric wards. Psychiatry Res. 2014;215(1):127-33.

34. Furre A, Sandvik L, Heyerdahl S, Friis S, Knutzen M, Hanssen-Bauer K. Characteristics of adolescents subjected to restraint in acute psychiatric units in Norway: a case-control study. Psychiatr Serv. 2014;65(11):1367-72.

35. Leidy BD, Haugaard JJ, Nunno MA, Kwartner JK. Review of restraint data in a residential treatment center for adolescent females. Child Youth Care Forum. 2006:35(5-6):339-52.

36. Donovan A, Plant R, Peller A, Siegel L, Martin A. Two-year trends in the use of seclusion and restraint among psychiatrically hospitalized youths. Psychiatr Serv. 2003;54(7):987-93.

37. Hottinen A, Valimaki M, Sailas E, Putkonen H, Joffe G, Puukka P, et al. Mechanical restraint in adolescent psychiatry: a finnish register study. Nord J Psychiatry. 2013;67(2):132-9. 